\title{
Mosaic trisomy 17
}

INSERM

\section{Source}

INSERM. (1999). Orphanet: an online rare disease and orphan drug data base. Mosaic trisomy 17. ORPHA:1711

Mosaic trisomy 17 is a rare chromosomal anomaly syndrome, with a highly variable clinical presentation, mostly characterized by growth delay, intellectual disability, body asymmetry with leg length differentiation, scoliosis, and cong enital heart anomalies (e.g. ventricular septal defect). Prenatal ultrasound findings include intrauterine growth retardation, nuchal thickening brain anomalies (e.g. cerebellar hypoplasia), pleural effusion and single umbilical artery. Patients with no associated malformations have also been reported. 